\title{
Kommunikation, Sprache und das Inklusionsproblem der Umweltethik
}

\author{
Konrad Ott, Christian-Albrechts-Universität zu Kiel
}

\begin{abstract}
Summary. This article contributes to solving the demarcation problem in environmental ethics. From within a discourse-ethical framework a criterion for the "capability to communicate" is proposed. After presenting some paradigm examples of communication within the non-human biotic world, four layers of communication are distinguished. Two layers occur in the non-human world, two remain specific to humans ("speaking a language by using speech acts" and "discourse"). It is argued that vocal and vocal-gesture communication is of moral relevance. Finally, three criteria for moral considerability are compared: "sentience", "interests", and "capability to communicate". This comparison points to parallels and implies a proposal of how to deal with the demarcation problem.
\end{abstract}

Zusammenfassung. Der Artikel liefert einen Beitrag zur Lösung des Inklusionsproblems der Umweltethik. Ausgehend von einer diskursethischen Rahmenkonzeption wird „Kommunikationsfähigkeit“ als Kriterium direkter moralischer Brücksichtigungswürdigkeit eingeführt. Es werden im Anschluss an einige Beispiele für Kommunikation in der außermenschlichen Natur vier Kommunikationsebenen unterschieden, von denen zwei in der außermenschlichen Natur vorkommen und zwei humanspezisch sind (in Sprechakten eine Sprache sprechen, Diskurs). Die Ebene der lautlichen und lautgestischen Kommunikation wird als moralisch relevant ausgewiesen. Zuletzt erfolgt ein Vergleich der drei in sich unterschiedlich definierbaren Kriterien „Empfindungsfähigkeit“, „Interesse“ und „Kommunikationsfähigkeit“. Dieser Vergleich zeigt parallele Strukturen auf und führt zu einem Vorschlag zum Umgang mit dem Inklusionsproblem.

\section{Das Inklusionsproblem und Vorschläge zu seiner Lösung}

Der nachfolgende Artikel ist aus einem umweltethischen Interesse heraus verfasst. Es wird überlegt, ob eine Analyse der verschiedenen Formen der Kommunikationsfähigkeit außermenschlicher Naturwesen dazu beitragen kann, das sogenannte Inklusionsproblem zu lösen. Als „Inklusionsproblem“ 
bezeichnet man die Frage, ob, und wenn ja, welchen Naturwesen ein inhärenter moralischer Selbstwert (synonym: „intrinsischer Eigenwert“) zuerkannt werden soll. Diese Zuerkennung impliziert die Aufnahme der betreffenden Wesen in die moral community und die Übernahme von direkten moralischen Verpflichtungen den betreffenden Wesen gegenüber. Eine (meta)ethische Analyse des Inklusionsproblems habe ich an anderer Stelle vorgenommen (Ott 2008). Die in der Umweltethik maßgeblich vetretenen Positionen (Sentientismus, Biozentrik, Ökozentrik, Holismus) werden als bekannt vorausgesetzt.

Das Inklusionsproblem verweist auf $\mathrm{Kr}$ i t e ri e $\mathrm{n}$, die den Ein- und den Ausschluss von Wesen in die moral community regeln, also in einem logischen Sinne zwischen unterschiedlichen Wesen diskriminieren. Genau darin liegt ja die Funktion von Kriterien: Unterschiede sichtbar zu machen. Die meistdiskutierten Kriterien zur Lösung des Inklusionsproblems waren und sind „Empfindungsfähigkeit", „Bewusstsein“ und „Interessen“. Empfindungsfähigkeit wird in allen Varianten des Sentientismus als Kriterium zugrundegelegt. Welche Wesen tatsächlich wie empfindungsfähig sind, wird als eine offene empirische Frage behandelt, für die die Biologie zuständig ist, wenngleich diese auch an begriffliche Einsichten gebunden ist.

Wenn Bewusstsein eine notwendige Voraussetzung für Empfindungsfähigkeit ist, und wenn Bewusstsein an ein zentrales Nervensystem gebunden ist, so sind alle Wirbeltiere (wahrscheinlich) empfindungsfähig. Das Bewusstsein von Fischen lohnt einen genauen Blick. Käfern, Spinnen, Bienen, Ameisen kann man aufgrund ihres organischen Bauplans allenfalls ein „(spürendes) Gewahren“ (mit Whitehead: „Prähensionen“) zusprechen, das einen abgeschwächten moralischen Status konstituieren könnte (Ott 2008). Der Interess e n b e griff lässt sich in einer schwächeren und in einer stärkeren Variante vertreten. In der stärkeren Variante verlangt er das „Haben“ von Interessen (,Die Kuh hat ein Interesse an Heu“), während es in der schwächeren Variante ausreicht, dass eine Handlung ,im Interesse“ eines Wesens ist („Es ist im Interesse der Pflanze, gewässert zu werden"). Der Unterschied zwischen beiden Varianten ist, grob gesagt, der zwischen Sentientismus und Biozentrik. Der begrifflich-kriteriale Verbund aus Empfindungsfähigkeit, die an Bewusstsein als an ihre Voraussetzung gebunden ist, und dem starken Interessenbegriff führt zum Sentientismus als derjenigen Lösung des Inklusionsproblems, der sich viele Ethiker*innen angeschlossen haben (L. Nelson, P. Singer, T. Regan, U. Wolf, A. Krebs, D. DeGrazia, C. Palmer und viele andere).

Das Problem der Abschwächung hat einen epistemischen und einen moralischen Aspekt: In epistemischer Hinsicht muss man fragen, ob etwa Bewusstsein als ein binär codiertes oder als ein graduell abgestuftes Phänomen auftritt. In moralischer Hinsicht fragt sich, ob graduelle Phänomene auch eine Hierarchisierung innerhalb der moral community erlauben. Wenn es beispielsweise so etwas wie ein dauerhaftes „DämmerBewusstsein" gäbe, so könnte der moralische Status entsprechender 
Wesen abgestuft werden. Der Streit zwischen egalitären und gradualistischen (= hierarchischen) Varianten innerhalb des Sentientismus wird im Folgenden ausgeblendet, da eine andere Fragestellung verfolgt werden soll.

Gesetzt einmal, man könnte zusätzlich zu dem kriterialen Verbund aus Empfindungsfähigkeit und Interessen a) (mindestens) ein weiteres Kriterium zur Lösung des Inklusionsproblems einfügen und b) die hieraus sich ergebenden Resultate mit dem Sentientismus vergleichen. Würde man nun unter diesem weiteren Kriterium zu gleichen Lösungen des Inklusionsproblems gelangen, so hätte man einen Grund zu der Vermutung, dass diese Lösung nicht völlig verfehlt (oder sogar „richtig“ bzw. „vernünftig“) sein könnte. Würde man hingegen zu völlig anderen Resultaten gelangen, so ergäben sich kompliziertere Folgeprobleme, die uns aber erst dann interessieren müssen, wenn dieser Fall einträte.

Rein logisch ist es möglich, dass zur Lösung des Inklusionsproblems „kein“, „genau ein“, „mehr als nur ein“ und „(beliebig oder unendlich) viele“ Kriterien herangezogen werden dürfen. Wer Kriterien ablehnt, wie T. Birch (1993) und M. Gorke (2010) dies tun, vertritt die Lösung „kein Kriterium“ und wird dann zum Holisten. Die einzige Grenze der moralischen Berücksichtigung stellt sich dann bei der Frage, welche Wesen es überhaupt "gibt", das heißt, welchen Entitäten der Existenzprädikator zukommt. Existieren Wellen und Zahlen? Wer das Inklusionsproblem für unlösbar hält, vertritt die Lösung „(beliebig oder unendlich) viele“. ${ }^{1}$

Ich vertrete und verfolge die Hypothese, dass mehr als nur ein Kriterium, aber nicht beliebig viele Kriterien sinnvoll sind. Ich ziehe im Folgenden ve r s u ch s we i s e das Kriterium „Kommunikationsfähigkeit“ heran, das sich aus einer diskursethischen Perspektive nahelegt. Dieses Kriterium besagt, dass Wesen, die mit anderen Wesen der eigenen oder anderer Spezies eine „wirkliche“ kommunikative Beziehung aufnehmen können, um ihrer selbst willen moralisch zu berücksichtigen sind. Dieses Kriterium verlangt eine begriffliche Analyse. Ich werde zunächst die diskursethische Perspektive erläutern (2), dann einige Beispiele für nicht-menschliche Kommunikation diskutieren (3), um ein kommunikationstheoretisches Ebenenmodell einführen (4) und mit Blick auf menschliche Sprachleistungen erläutern zu können (5). Im Anschluss diskutiere ich mögliche Lösungen des Inklusionsproblems unter diesem Kriterium (6), um diese Lösungen abschlieBend mit den anderen Kriterien zu vergleichen (7).

\section{Diskursethik und Kommunikationsfähigkeit}

Die Diskursethik geht von dem Gedanken aus, dass eine an Kant anschlieBende Begründung von Moralprinzipien das theoretische Design einer Sprachethik annehmen muss. Dieser Gedanke stützt sich auf den Übergang zur Sprachphilosophie (sog. linguistic turn), der sich am Beginn des 20. Jahrhunderts vollzieht und in dessen Verlauf durchsetzt. Die eigentli- 
che Begründung moralischer Prinzipien erfolgt bei Apel (1976) in der Auseinandersetzung mit dem Fallibilismus der Popper-Schule und dem sogenannten Münchhausen-Trilemma, das eine Begründung oberster Prinzipien ausschließt, sofern sie als Ableitung konzipiert wird. Die transzendentalpragmatische Reflexion auf Voraussetzungen sinnvoller Rede soll bei Apel zu den Handlungsprinzipien führen, die realen Kommunikationsgemeinschaften zu erhalten und auf die Beförderung einer idealen Sprachgemeinschaft in den realen hinzuwirken. Habermas (1983) möchte demgegenüber nur ein Diskursprinzip normativer Gültigkeit aus Diskursregeln ableiten. Diese Begründungen sind seit den 1980er Jahren Gegenstand einer umfänglichen ethischen Debatte, die hier nicht ausgebreitet werden kann (Gottschalk-Mazouz 2000).

Seit dieser Zeit ist auch versucht worden, dieses ethische Rahmenkonzept, das zunächst nur die diversen commitments of arguing expliziert und alle substantielle Normen zu Gegenständen spezifischer Diskurse erklärt, auf den Bereich der Umweltethik anzuwenden. So hat John Dryzek (1990) schon früh dafür plädiert, die kommunikativen Akte von Naturwesen mittels einer advokatorischen Sprecherrolle zu versprachlichen, um sie sie so als indirekte Beiträge zu praktischen Diskursen und umweltpolitischen Debatten zulassen zu können. Ähnliche Überlegungen finden sich auch in meinen eigenen frühen Arbeiten (Ott 1993). Vor allem schienen mir tierische Schmerzenslaute geeignet, als Nein-Stellungnahmen interpretiert zu werden. Dies führte mich zu der Idee einer möglichen $\mathrm{Path}$ o g n o mik der Natur, die ich auf alle Lebewesen und sogar auf Ökosysteme ausweiten wollte. Diese frühen Versuche, ein Kriterium „Kommunikationsfähigkeit“ für die Umweltethik fruchtbar zu machen, wurden allerdings nicht systematisch weiterverfolgt, da sie auf harsche Kritik innerhalb des diskurstheoretischen Paradigmas stießen.

Stattdessen wurde ein anderer Weg eingeschlagen, um Diskurs- und Umweltethik systematisch aufeinander zu beziehen. Die Diskursethik fordert konzeptionell in jedem Falle eine umfassende epistemische, phänomenologische, axiologische und deontologische Bestimmung von unterschiedlichen Mensch-Natur-Verhältnissen. Vermutlich wurde, wenn man Habermas (1984) folgt, das Vernunftpotential der naturbezogenen Rede in einer von szientistischen, technologischen und ökonomisierten Denkformen beherrschten Moderne nur höchst selektiv ausgeschöpft. Nur solche Rede über Natur sollte nach modernistischem Dafürhalten als rational gelten, die sich auf eine objektiv erklärbare, eine technologisch manipulierbare oder eine ökonomisch bewertbare Natur bezögen. Allenfalls zugelassen wurden naturästhetische Äußerungsformen, die allerdings als ,romantisch“ galten. Die Angst vor einer „Wiederverzauberung der Natur", die sich auch bei Habermas findet, schnürte viele naturbezogene Sprachformen ab. Eine diskursethisch verankerte Umweltethik ließ sich als Versuch verstehen, das Artikulationsspektrum auf allen Ebenen der zwischenmenschlichen Kommunikation über Natur und Umwelt zu erweitern und zu vertiefen (Ott 2010). Der Diskurs des Umwelt-, Tier- und Naturschutzes sollte, gleichsam im Geiste kritisch- 
skeptischer Solidarität mit den Anliegen dieser Schutzbewegungen, diskursrational rekonstruiert werden.

Auch wir Menschen kommunizieren durch Laute und Lautgesten über die Art, wie sich Natur zeigt. So eröffnet sich das sprachliche Vernunftpotential naturbezogener Rede bei lautlichen, häufig von Gesten begleiteten Evokationen wie „Großartig!“”, „Wie schön!“, „Herrlich!“. Auch Narrative und Schilderungen, die Naturerfahrungen erhalten, sind wichtig zur Verständigung über Naturwerte. Das Genre des nature essay ist hier ebenfalls zu erwähnen. Artikulation von Naturerfahrungen können auch in Sprichworten, Liedern und Gedichten aufbewahrt werden, die es zu überliefern lohnt. Eine diskursive Ebene der Auseinandersetzung wird erreicht, wenn naturschützerische Intuitionen (Artenschutz, Biodiversitätserhalt, Wildnisschutz, Renaturierungsmaßnahmen) gegenüber skeptischen Opponenten in allgemein anerkennenswerte Gründe übersetzt werden sollen. Durch solche Gründe wird niemand emotional zum Naturschutz motiviert, aber kognitiv verpflichtet, die Anliegen des Umwelt-, Tier- und Naturschutzes als legitim zu respektieren. Diese diskursive Arbeit beginnt, so gesehen, auf einer tabula rasa bzw. einem leeren Feld, in das sukzessive Gründe eingetragen werden können, die für die Anliegen des Umwelt-, Tier- und Naturschutzes sprechen. Durch diese Eintragungen bildet sich ein spezifisches universe of environmetal discourse heraus. Dieses Diskursuniversum wird auch als Argumentationsraum bezeichnet (Ott 2010). Umweltethik besteht im „Durchlaufen" (,discurrere" = , durchlaufen") der einzelnen Gründe, die gleichsam „Stationen“ des Diskurses sind. Naturwesen haben keinen direkten Zugang zu diesem Argumentationsraum, der daher in epistemischer Hinsicht anthropozentrisch ist. Der anthropos ist jetzt allerdings idealiter eine Person geworden, die diesen Argumentationsraum durchlaufen und seine Inhalte kritisch geprüft und sie sich angeeignet, d.h. begriffen hat. Diese Prüfung schließt das Inklusionsproblem ein.

Die Diskursethik ist nicht auf eine anthropozentrische Lösung des Inklusionsproblems festgelegt (Werner 2003). Wie Hendlin und Ott (2016) ausführlich gezeigt haben, pendelt Habermas selbst zwischen starken physiozentrischen Intuitionen und einer metaethisch motivierten Zurückhaltung hin und her, von der Hendlin und Ott glauben zeigen zu können, das sie unbegründet ist. Die Auseinandersetzung mit Habermas' impliziter Umweltethik zeigt, dass es sich lohnen könnte, noch einmal genauer auf das Kriterium der Kommunikationsfähigkeit zurückzukommen. Hierfür sprechen neben spezifisch diskursethischen Überlegungen auch die Fortschritte in den Forschungen über Tier,,sprachen" und in der allgemeinen Biosemiotik (Barbieri 2008).

\section{Beispiele für kommunikatives Verhalten von Naturwesen}

Die Ausdrücke „Kommunikation“ und „Sprache“ sollten nicht willkürlich („stipulativ“) definiert werden. Womöglich zählen sie sogar zu den essentially 
contested concepts, d.h. zu Konzepten, die man nur versteht, wenn man weiß, warum sie in diversen Zweigen der Wissenschaft (Biologie, Linguistik, Philosophie, Informatik) umstritten sein müssen. Auf den Begriff der Sprache komme ich weiter unten ausführlich zurück; hier geht es zunächst um den Begriff der Kommunikation und um dessen dispositionelles Gegenstück, die Kommunikations fä h i g k e i t . Ein Wesen ist kommunikationsfähig genau dann, wenn es (performativ) Leistungen zu vollbringen vermag, die unter den Begriff der Kommunikation fallen. Dann „kommuniziert“ es. Eine Annahme, wonach alles Verhalten Kommunikation „sei“, erscheint begging the question. Kommuniziert die Schlange mit dem Frosch, den sie verschlingt? Kommunizieren Parasiten mit ihrem Wirtsorganismus? Diese Annahme wäre eine überbestimmende Gleichsetzung.

Mittlerweile dürften die meisten Biologen und viele Philosophen zu der Ansicht neigen, dass viele Naturwesen (miteinander) k o m m u n i z i e r e $\mathrm{n}$. Umstritten ist, ob es sich hierbei (nicht) um sprachliche Kommunikation handelt. Die Qualifikation einer Kommunikation als „sprachlich“ setzt bereits eine differentia specifica, die einen Sprachbegriff voraussetzt (Abschnitt 4). Me th o d i s ch ist es zunächst sinnvoll, einen weiten Begriff der Kommunikation einzuführen, um den Phänomenbereich umschreiben, Beispiele einführen und späterhin den wesentlichen Unterschieden Rechnung tragen zu können. Im weitesten Sinne bedeutet Kommunikation zunächst, dass Informationen übermittelt werden.

Wenn man das technomorph konzipierte Modell „Sender-Kanal/Medium-Empfänger" auf Organismen überträgt, so kann man immer dann von Kommunikation zwischen Naturwesen sprechen, wenn die Information des Sender-Organismus eine beobachtbare, zumeist zeitlich verzögerte Auswirkung auf das Verhalten des Empfänger-Organismus hat. Diese Art der Kommunikation qua Informationsübermittlung findet nun auch bei Pflanzen statt (Trewavas 2014). Es ist möglich, vielen Organismen eine kommunikative Fähigkeit zur Semiose, d.h. zur Zeichenproduktion und zur Informationsübertragung zuzuerkennen, der Fähigkeiten anderer Organismus korrespondieren, diese Information zu „Iesen“ (dechiffrieren, deuten, verstehen). Der Begriff der Information ist dabei ein sinnvoller Begriff in der theoretischen Biologie (Maynard Smith). Die Übermittlung von Information durch biochemische Signale, wie sie zwischen Pflanzen auch unterschiedlicher Spezies stattfindet, kann in diesem Sinne als „plant communication“ bezeichnet werden (Trewavas 2014). ${ }^{2}$

Manche Verhaltensweisen sozialer Insekten, etwa die Tänze der Bienen, deren körperliche Bewegungen Botschaften über Futterplätze an andere Bienen übermitteln, lassen sich als innerartliches informationsübermittelndes und kommunikatives Handeln verstehen. Der Unterschied zu Pflanzen liegt (vermutlich) darin, dass die „tanzende“ Biene in der artspezifischen Umwelt der anderen Bienen (als Artgenossin) präsent ist. Ein solcher Bewegungsablauf (der anthropomorph als „Tanz" bezeichnet wird) versetzt nämlich andere Bienen in die Lage, auch ohne die Botschafterin den Weg zur angezeigten Futterstelle zu finden. Die Körperbewegungen 
einer Biene können somit von anderen Bienen als (beabsichtigte) I n for m a ti o n süb e r m it t l ung verstanden werden. Es wird von einem Organismus anderen Organismen etwas Informatives mitgeteilt, das das Verhalten dieser Organismen beeinflusst. Adaptives Ziel ist das foraging des gesamten Bienenstocks. Zwar hat Bennet (1967) den Bienentänzen den Charakter der vollwertigen Sprachlichkeit abgesprochen und innen nur Sprach ä h $\mathrm{n}$ l i c h k e it zugebilligt. Dass es sich um Kommunikation handelt, ist jedoch unbestritten.

Tiere sind nun beweglich und allotroph. Der Mund-Anus-Trakt bei Tieren ist in Verbindung mit dem zentralen Nervensystem ein anderer „Bauplan" als der Wurzel-Röhre-Blatt-Trakt der Pflanzen. Der Mund-Anus-Trakt vieler Tiere setzt den Mund soweit "frei“, dass er Laute generieren kann. Daher ist das Tierreich auch ein Reich der kommunikativen Lautgaben. Die cartesianische Auffassung, es handele sich bei diesen Lauten um bloße Geräusche, ist nicht zu halten. Die Übermittlung von Information bleibt in diesen Lautgaben mit vorausgesetzt, nimmt aber eine andere Gestalt an.

Sogenannte Warnrufe von Vögeln, die beim Herannahmen von Beutegreifern ausgestoßen werden, sind kommunikative Akte. Es sind Ruflaute, die situationsspezifisch an Artgenossen adressiert sind und einen Adaptionswert im Sinne von inclusive fitness einer genetisch verwandten Population aufweisen müssen. Forschungen an Affen haben gezeigt, dass Affen andere Affen vor unterschiedlichen Prädatoren warnen können. Die Warnungen differenzieren lautlich unterschiedliche Gefahrenquellen (,Schlange im Gebüsch'versus ,Adler aus der Luft') (Hauser 2001: 236ff. mit Verweis auf diverse empirische Studien).

Manche verhaltensförmigen kommunikativen Sachverhalte sind rein evolutionär schwer erklärbar. Wenn beispielsweise ein Hase sich in sicherer Entfernung dem anschleichenden Fuchs „zeigt“, ihm also zu „verstehen“ gibt, besser von der erfolglosen Jagd abzulassen, dann fragt sich, welche zoologische Theorie uns zu der besten Erklärung dessen verhilft, was sich unter Tieren ereignet. „Weiß“ der Hase, dass der Fuchs „weiß“, dass er die energetisch aufwändige Jagd verliert, wenn er nicht nahe genug an Hasen heranpirschen kann? Wenn ja, warum sollte ein Beutetier irgend einen adaptiven Vorteil davon haben, seinem Prädator die Jagd zu ersparen?

Dass das Jagdverhalten von Tiergruppen mit kommunikativen Akten durchsetzt ist, ist empirisch schwer zu bestreiten. Ähnliches gilt für Balz-, Brutpflege- und Herdenverhalten. Kommunikative Akte erfolgen, wenn Affen anderen Affen beibringen, Knollen vor dem Verzehr zu waschen, was deren Geschmack verbessert und die Zähne der Affen schont. Es wäre interessant, lautliche Kommunikation unter der Perspektive des sog. Baldwin-Effektes zu analysieren, wie Hoffmeyer und Kull (2003) dies für die Biosemiose tun. Manche Tiere vermögen innerhalb ihrer Spezies und sogar über Artgrenzen hinweg durch Vokalisation miteinander zu kommunizieren, wobei offenbar sogar sogenannte audience effects zu beobachten sind, d.h. Wirkungen auf anwesende Tiere, die nicht direkt an der Kommunikation beteiligt sind (Fichtel und Manser 2010). Dass Jagdverhalten von Rudeltieren 
mit kommunikativen Akten durchsetzt ist, ist schwer zu bestreiten. Ähnliches gilt für Balz- und Brutpflege- oder Herdenverhalten. Umstritten ist, ob tierische Kommunikation bis hin zu intersubjektiver Handlungskoordination reicht. Ist die von kommunikativen Akten begleitete Jagd der Schimpansen eine gemeinsame Jagd oder bleibt jeder einzelne Schimpanse bei diesen Jagden in der monologischen Perspektive befangen? Letzteres behauptet Tomasello, dem sich Habermas in dieser Frage anschließt. Haben Prädatoren, die kollektiv jagen, ein gemeinsam geteiltes Verständnis von ihrer Aktivität, d.h. eine Vorstellung davon, dass sie „miteinander im Rudel“ jagen? Hendlin und Ott (2016) zeigen, dass Tomasellos Deutung keineswegs alternativenlos ist. Wir möchten höheren Tieren also die Fähigkeit zur Handlungskoordination durch lautliche und lautgestische Kommunikation nicht rundweg absprechen.

In lautlichen Selbstbekundungen demonstrieren höhere Tiere ihre „Präsenz" (Elefanten, Kraniche). Der Vogelgesang ist von kommunikativer und expressiver Art. Zur Artikulation von La u tg e s te $n$ sind viele Säugetiere in der Lage. Auch Meeressäuger kommunizieren lautlich miteinander (Simmonds 2006: 108f.; King und Janik 2013). Delphine identifizieren Miglieder ihres Verbandes individuell durch bestimmte Töne, die einem Eigennamen zumindest analog sind. Auch der gemeinsame Drogenkonsum der Delphine, die nacheinander Kugelfische „abschlecken“, ist eine kommunikative Interaktion. Die „Gesänge“ der Wale dienen dazu, einander in den Weiten des Ozeans zu begegnen, sind also zugleich adaptiv und kommunikativ.

Lautgesten sind kommunikative Gebärden und es ist bekannt, dass George H. Mead - und vor inm schon Wilhelm Wundt - aus Lautgestenkommunikation heraus die Entstehung der menschlichen Sprache herleiten wollte. Der Weg von vokalen Ausrufen über Lautgesten zu signifikanten Symbolen ist für Mead (1973: 100-107) zwar lang; gleichwohl kann man Lautgestenkommunikation als genuin protosprachlich auffassen, sofern dieser Weg immanent angelegt ist, aber bestimmte Tierarten zu unterschiedlichen Punkten auf diesem „Weg zur Sprache“ gelangen. Es erscheint uns Menschen möglich, einzelne Tiere durch Lernangebote auf diesem Weg weiterzubringen (siehe unten). Das „Protos“ dieser Sprachlichkeit ist eigens zu bedenken (siehe unten).

Am dichtesten an Sprachlichkeit heran reicht zweifellos die Kommunikationsfähigkeit der Primaten, insbesondere der Schimpansen und Bonobos. Menschen können mit Primaten, denen man eine Zeichensprache beigebracht hat, in einen komplexen sprachlichen Austausch eintreten (Russon u.a. 1996). Hierdurch ist offenbar auch ein sprachlicher Austausch über Gefühle wie Trauer möglich, die eine Äffin empfindet, wenn man ihr einen Todesfall mitteilt (Gardner u.a. 1989). Dies scheint eine wirkliche sprachliche Verständigung über Artgrenzen hinweg zu sein. Primaten können sprachlich kommunizieren, wenn Menschen ihnen eine (einfache) Bilderund Gestensprache nahe- und beibringen. Die Tiere sind intelligent und bereits der Lautgestenkommunikation fähig; daher kann man innen die Bil- 
der- und Gestensprache beibringen, die ursprünglich zur sprachlichen Kommunikation unter und mit Menschen dienen sollen, die ein organismisch bedingtes "Handicap“ haben, also taubstumm sind. Dies ist ein upgrading in den Bereich der Sprache, das von uns Menschen intentional veranlasst und von Tieren vollzogen wird.

\section{Kommunikationsebenen}

Es gibt, wie ansatzweise gezeigt, allerdings unterschiedliche Arten von Kommunikation. Extension (Umfang) und Intension (Bedeutung) von Begriffen stehen bekanntlich im gegenläufigen Verhältnis: Wird die Extension gröBer, wird die Intension geringer und umgekehrt. Etwas wird seltener und anspruchsvoller: Wenn jeder mein Freund ist, mit dem ich eine Visitenkarte ausgetauscht habe, gibt es viele Freundschaften, aber Freundschaft bedeutet fast nichts mehr. Wenn nur diejenigen meine Freunde sind, die für mich zu sterben bereit sind, habe ich vielleicht keinen einzigen Freund, aber Freundschaft wird zu einer höchst bedeutsamen zwischenmenschlichen Beziehung. Dieses Verhältnis lässt sich mutatis mutandis als Verhältnis unterschiedlicher kommunikativer Ebenen konzipieren, wobei auf den höher bezifferten Ebenen die Extension abnimmt und die Intension anwächst, d.h. eine bestimmte Art der Kommunikation wird seltener und anspruchsvoller. Ich unterscheide folgende vier Ebenen:

1. Informationsübermittlung,

2. Lautliche Kommunikation ${ }^{3}$,

3. Sprechhandeln, „eine Sprache sprechen“,

4. Diskursivität, Argumentation.

Jede nachfolgende Ebene schliesst die früheren ein. Auch in Diskursen kommt es zur Übermittlung von Informationen, die an Gründe anknüpfen. So können neue Informationen, die sprachlich als Tatsachen repräsentiert werden (,Es ist der Fall, dass p“) bestimmte etablierte Gründe entwerten oder stärken. Wer auf der Grundlage falscher Informationen argumentiert, muss revozieren usw. Wir argumentieren auf einer bestimmten wandelbaren Informationsbasis, die bereits sprachlich repräsentiert ist.

Der weite Begriff der (bio)semiotischen Informationsübermittlung bzw. -übertragung kann auf der zweiten Ebene zu einem Begriff der lautlichen Kommunikation eingeschränkt werden, der viele Tiere einschließt und auf das beiderseitige Verständnis eines Miteinanders bezogen wird. Tierische Kommunikation auf Grundlage lautlicher Bekundungen kann innerhalb des Modells uneingeschränkt zugelassen und gewürdigt werden. Die Welt der Tiere ist, wie im vorigen Abschnitt ausgeführt wurde, keine Welt bloßer Geräusche, sondern umschließt leiblich gebundene Lautgaben, die bis hin zu stimmlicher Artikulation und Protosprache reichen können. Die Lautkommunikation vieler Tiere liegt somit in einem Zwischenbereich zwischen 
Informationsübermittlung und dem „Sprechen einer Sprache“, dem ich mich nun zuwende.

\section{Leistungen der menschlichen Sprache}

Wird der Begriff der S p ra c h e so bestimmt, dass nur Menschen Sprachwesen sind, so argwöhnen viele Umweltethiker*innen eine hochnäsige Selbstprivilegierung des Menschen, die mit dem moralischen Vorwurf des "Speziesismus" gebrandmarkt wird. Diese wohlfeile Art der Moralisierung enthebt jeglicher Anstrengung, den Begriff der Sprache zu bilden. Moralismus droht, im Begriff der Kommunikation alle Unterschiede zu nivellieren, weil man ja, wie es im Modejargon philosophischer Halbbildung (allzu) häufig heißt, nicht willkürlich sein dürfe. Der Willkürvorwurf lässt sich gegen jedes differenzierte Denken leicht erheben; er macht der gedanklichen Faulheit und der begrifflichen Bequemlichkeit ein gutes moralisches Gewissen.

Im Unterschied zu Informationsübertragung und zu lautlicher oder gestischer Kommunikation scheint sprachliche Kommunikation vorauszusetzen, dass ein Wesen eine (bestimmte) Sprache spricht. Lautliche Kundgabe und Lautgesten wären demnach eo ipso noch nicht sprachlich, sofern eine Sprache (im Sinne Saussures) eine Art von System aus bedeutungshaltigen Zeichen wäre, die sich syntaktisch und grammatisch kombinieren ließen. Die tierischen Lautgaben greifen nicht auf solch ein System (Saussure: langue) zurück. In der Lautkommunikation ist die Bedeutung unmittelbar gegeben; im Sprechen einer Sprache lösen sich die Bedeutungen von der Unmittelbarkeit der Situationen und es entstehen hierdurch weitere emergente bzw. symbolische Leistungen, die nur "in“ einer Sprache möglich sind (umfassend hierzu Westerkamp 2016).

Diese Bestimmung schließt echte Tiersprachen (etwa bei Walen und Delphinen) nicht aus - zumindest dann nicht, wenn man echte Sprachen zuließe, die (fast) nur aus Einwortsätzen bestünden. Von diesem Fall ist freilich der Fall zu unterscheiden, dass einzelne Tiere eine von Menschen entwickelte Zeichen- oder Bildersprache zu lernen in der Lage sind. Dies wäre keine „Tiersprache“, obschon die betreffenden Tiere in gewisser Weise zu Sprachwesen erhöht werden, wenn man ihnen diese Sprache beibringt. Und nur weil man innen Sprache beibringen kann, sind diese Tiere in genau diesem Sinne sprachfähig. Wir Menschen ermöglichen einzelnen besonders begabten Tieren gleichsam erste Schritte in die Welt der Sprache.

Der Begriff der Sprache lässt sich so verstehen, dass „eine Sprache sprechen" e rsten s bedeutet, in der Rede (parole) auf ein System von bedeutsamen Zeichen (langue) zurückgreifen zu können, dass es ermöglicht, von beschränkten Mitteln einen fast unbegrenzten Gebrauch machen zu können. Die Rede ist dabei z we it e n s immer Wechselrede, d.h. eine Unterredung von mindestens zwei Sprecher*innen, die in der Rede auf das System der Personalpronomina zurückgreifen können. ${ }^{4}$ Man kann bei der 
Bestimmung des Begriffs der Sprache dritten s auch auf die Rekursivität und Reflexivität von Sprachlichkeit und, vi e rtens, auf das eigenartige Verhältnis zwischen den vielen Einzelsprachen und der in ihnen beschlossenen sprachlichen Weltbilder (von Humboldt 1829) und der gleichwohl zwischen innen möglichen Übersetzbarkeit verweisen (Hönigswald 1937). Wer eine bestimmte Sprache spricht, spricht immer zugleich "die“ Sprache (Hönigswald 1937). Die Lautgaben der Krähen und der Katzen ließen sich, so eine Hypothese, nicht so ineinander übersetzen wie das Englische sich ins Japanische übersetzen lässt. ${ }^{5}$

Die genuine Sprache ist auf der Ebene der Sprechhandlungen, d.h. des Sprechens einer Sprache erreicht. Dieses Sprechen ist mit dem Erheben von Geltungsansprüchen in Sprechhandlungen wesentlich verbunden. Die Geltungsmodi der menschlichen Sprache sind vielfältig. Vollständige Sprechakte sind beispielsweise Versprechen, Warnungen, Ratschläge, Bitten, Fragen usw. Sprechakte bestehen aus einem performativen Teil, der den Status des Sprechaktes bestimmt („Ich bitte dich nicht, sondern fordere dich auf, x zu tun") und einem propositionalen Teil, der das x-Tun bestimmt. Menschen sind in der Lage, den Status ihrer Sprechakte zu variieren: „Das war nicht als Kritik, sondern als Lob gemeint!“ - „Ich konzediere dir x und ziehe deshalb meine Forderung zurück." Sprechakte haben illokutionäre Bindekräfte. Wer beispielsweise etwas als wahr behauptet, erwirbt damit Berechtigungen und Verpflichtungen. Wer behauptet, dass der Ball rot ist, ist verpflichtet, der Aussage zuzustimmen, der Ball sei farbig (zum Inferentialismus vgl. umfassend Brandom 1994). Mit Sprechakten eng verbunden ist die vierte Ebene, in der Geltungsansprüche expliziert und damit kritisierbar werden. So können Behauptungen g e I t u n g s l o g i s ch differenziert werden: „Ich behaupte als wahr gegenüber jedermann, dass p“ bedeutet etwas anderes als zu sagen: „Ich möchte zu bedenken geben, ob und inwiefern diese neue Information unsere bisherige Situationsdeutung verändert." Die Geltungssphäre der menschlichen Rede hat Habermas (1981) umfassend rekonstruiert. Aufgrund unterschiedlicher Weltbezüge der Rede können wir uns demnach in drei Geltungsdimensionen bewegen, denen ein unspezifischer Geltungsanspruch auf die Verständlichkeit einer Äußerung vorgelagert ist. Diese Dimensionen sind Wahrheit, Richtigkeit und Wahrhaftigkeit. Ein Sinn für unterschiedliche Geltungsmodi scheint bei Tieren zu fehlen. Unzugänglich ist den Tieren auch die reflexive Thematisierung von Geltungsmodi, die etwa erreicht ist, wenn wir uns beispielsweise fragen, welchen Geltungsbezug religiöse und theologische Rede eigentlich hat.

Man kann mit Sprechakten offene und verdeckte perlokutionäre Effekte erzielen wollen. Ein offener perlokutionärer Effekt besteht im Falle eines Befehls darin, den Adressaten zur Ausführung des Befehls zu motivieren. Ein verdeckter perlokutionärer Effekt besteht in einer Täuschung. Wenn beispielsweise A B von einer Handlung abrät, weil $A$ aus Eigeninteresse will, dass $B$ die Handlung unterlässt, dann macht sich $A$ die normative Bedeutung eines Ratschlags schlau zunutze. Wir unterstellen, dass Ratschläge im besten Interesse des Beratenen, nicht des Beratenden erfol- 
gen sollen. Unterstellungen und entsprechende Erwartungen sind die Bedingungen, aufgrund derer sich verdeckte perlokutionäre Effekte erzielen lassen (Habermas 1981; Ott 1997). Verdeckte perlokutionäre Effekte können auch mit moralischer Rede intendiert werden: Man kann mit moralischer Schmähkritik andere in ein schlechtes Licht rücken, demütigen oder verleumden. In ähnlicher Weise ist eine Intrige ein Zusammenspiel aus unterschiedlichen Sprachhandlungen mit dem Ziel, eine Person dazu zu bewegen, einer anderen Person zu schaden. Die Rhetorik kann als Kunstlehre hinsichtlich des Erzielens perlokutionärer Effekte (etwa Applaus) verstanden werden. Die menschliche Sprache eignet sich für Lug und Trug (und Sophistik), gerade weil es die menschliche ist.

Es gibt keine Anhaltspunkte, dass Naturwesen in ihrem lautlich-kommunikativen Verhalten die Ebene der Diskursivität erreichen. Sie wird nur dann erreicht, wenn Geltungsansprüche bestritten und mit Argumenten widerlegt oder verteidigt werden. Diskurse sind definiert als die Fortsetzung alltäglichen kommunikativen Handelns mit argumentativen Mitteln. In ihnen zählt idealiter nichts als der „zwanglose Zwang guter Gründe“, wobei sich die Güte von Gründen in Diskursen jeweils neu herausstellen muss, also vorab weder festgelegt werden kann noch darf. Die möglichen Übergänge vom kommunikativen Handeln zu Diskursen sind dabei in den Möglichkeiten angelegt, zu Geltungsansprüchen in Sprechakten des Bezweifelns, Bestreitens und Widerlegens ablehnend Stellung zu nehmen. Werden Geltungsansprüche kritisiert, eröffnet dies die Möglichkeiten, nach den jeweiligen Gründen „für und wider" zu fragen. In diesem Sinne sind „ich und du“, ,ja und nein" und „für und wider" gleichsam die Achsen, um die sich die Räder der Diskurse drehen.

Wer einen Geltungsanspruch argumentativ einlösen möchte, muss sie sich als Konklusion vorstellen, der die Prämissen fehlen. „Nach den Gründen zu einer Annahme gefragt, besinnt man sich auf diese Gründe" (Wittgenstein 1980, § 475, Hervorhebung im Original). Weil Gründe nachträglich gegeben werden dürfen, müssen Geltungsansprüche nicht immer abgeleitet worden sein. Dieses Sich-Besinnen-auf-Gründe ist Tieren offenbar nicht möglich. Die Einstellung, aus der heraus Diskurse geführt werden müssen, ist eine reflexiv-prüfende Einstellung zu Gründen aller Art. Diese sprachlogische Ebene der Diskursivität scheint für Naturwesen unerreichbar zu sein. Dies gilt auch für alle Arten geistiger Tätigkeit, die intern diskursiv verfaßt sind, wie Recht, Politik und Wissenschaft. Die sogenannte logos-Auszeichnung des Menschen bezieht sich daher auf die Ebenen 3 und 4, während wir die ersten beiden Ebenen mit Naturwesen teilen.

Auch wir Menschen können uns freilich in Momenten von Wut und Zorn in Wesen (zurück)verwandeln, die primär durch Lautgesten kommunizieren: Wir brüllen einander an und fuchteln drohend mit den Fäusten. Aus sprachlicher und diskursiver Perspektiven handelt es sich um Regressionen. Sanftere und "schönere“ Regressionen auf die lautgestische Ebene ereignen sich in der Geschlechtsliebe. In diesem Sinne ist uns Menschen diese lautgestische Ebene nicht fremd. 
Somit können wir im Anschluss an Ergebnisse linguistischer Forschungen einige Merkmale und Leistungen aller menschlicher Sprachen auf Ebene 3 und 4 identifizieren, die man mit einer Absentismus-Hypothese verknüpfen kann: Wir können sprachlich $\mathrm{x}$ tun und für das betreffende $\mathrm{x}$ gibt es keinerlei Belege bei tierischer Kommunikation. Wir Menschen kommunizieren untereinander zwar bei bestimmten Gelegenheiten auch durch Pheromone und durch Lautgesten, aber vor allem sprechen wir Sprachen, um uns in der Wechselrede über etwas zu verständigen (von Humboldt 1829; Hönigswald 1937; Habermas 1981). Menschliche Sprachen, wie immer ihre Entstehung evolutionär erklärt werden mag (Deacon 2003, Hauser u.a. 2002), weisen bestimmte Charakteristika auf, die lautlicher Komminikation fehlen: „Menschliche Normalsprachen sind semiotisch differenziell, syntaktisch rekursiv, semantisch reflexiv und pragmatisch inferentiell organisiert. Keine andere Kommunikationart übersteigt sich selbst zu etwas, das mehr ist als Informationsaustausch" (Westerkamp 2016: 119). Nur die menschliche Sprache weist eine starke Tendenz zur Schrift und damit zur „skripturalen Sinnerzeugung" (Westerkamp 2016: 121) auf. Menschliche Sprachen sind weiterhin temporal und modal differenziert. „Differenzialität, Rekursivität, Reflexivität und Inferentialität“ (Westerkamp 2016: 133) könnten, sofern sie theoretisch-konzeptionell integriert würden, ein starkes Argument für humanspezifische Sprachleistungen ergeben. Hinzu kommt noch die Fähigkeit zur Diskursivität. Sich mit Gründen an Gründen orientieren zu können, ergibt sich aus der Rekursivität von Sprache und der Möglichkeit, Sprechhandeln mit den Mitteln der Argumentation fortzusetzen. Dadurch bilden sich die Sphären des objektiven und des absoluten Geistes (sensu Hegel). Diese sind für Naturwesen unzugänglich.

\section{Zurück zum Inklusionsproblem}

Naturwesen sind auf je unterschiedliche Weise kommunikative Wesen, aber keine Sprachwesen im engeren Sinne. Aber was sollte in moralischer Perspektive für die Lösung des Inklusionsproblems eher „zählen“: das, was Naturwesen kommunikativ vermögen, oder das, wozu sie außerstande sind? An dieser kritischen Stelle der Argumentation schalte ich folgende Überlegung ein. Die Betonung genuin menschlicher Sprachleistungen kann mit einer Aufwertung nichtmenschlicher Kommunikation widerspruchsfrei einhergehen. Die Angst, durch Anerkennung und Aufwertung dieser kommunikativer Fähigkeiten die Grenze zwischen Menschen und Tieren zu verwischen, erweist sich nunmehr als unbegründet, da die logos-Auszeichnung auf Sprachebene 3 und 4 aufbewahrt ist. Die Aufwertung tierischer Kommunikation impliziert keine Nivellierung zwischen dieser und der menschlichen Sprache: Je besser wir nicht-menschliche Kommunikation verstehen und würdigen, um so höher können wir Diskursivität wertschätzen (und uns selbst, sofern wir von ihr Gebrauch machen, ihrer würdig erweisen). 
Schon die biochemische Signalübertragung bei Pflanzen ist von erstaunlicher Komplexität, wie die Beiträge in Baluška u.a. (2006) eindrücklich dokumentieren. Das „alte“ Bild der Pflanzen ist zu Recht im Umbruch begriffen, wobei auch davor gewarnt wird, Pflanzen unzulässig zu „zoomorphisieren“. Pflanzen sind auf sensorische Weise sensitiv, registrieren biochemische Signale und haben schwache Interessen. Es bleiben gleichwohl Zweifel, ob diese Eigenschaften für die Zuerkennung von moralischem Selbstwert hinreicht. Pflanzen können Informationen zwar austauschen, aber nicht miteinander kommunizieren, da sie nichts voneinander wissen (müssen). Die biochemischen Signale, so könnte man vielleicht sagen, „, schwirren“ im Pflanzenreich gewissermaßen anonym durch die Luft, ähnlich wie Sonnenstrahlen in bestimmten Einfallswinkeln. Solche Signale werden „aufgefangen“ und lösen vorprogammierte Reaktionen aus, wie dies auch bei automatischen Sensoren vorkommt. Man hält ein elektronisch codiertes Kärtchen an eine Stelle, es blinkt „grün“ und die Tür geht auf. In der technischen Welt können Signale programmiert und geschaltet werden. Pflanzen wären in diesem Sinne „sensorische“ Wesen, die stricto senso nicht m i t e in a n d e r kommunizieren. An diesem Punkt trete ich dafür ein, Pflanzen in ihrer Alterität zu Tieren ernst zu nehmen und sie nicht zu „zoomorphisieren“. Ein Naturwesen in seiner Besonderheit ernstnehmen, kann nun auch bedeuten, ihm ke i n e n Selbstwert zuzuerkennen: Daher ist der „Respekt“ Pflanzen gegenüber paradox: Ich respektiere Pflanzen als solche, indem ich innen moralischen Selbstwert abspreche. Die instrumentelle und die ästhetische Wertschätzung des Pflanzenreiches ist von diesen Überlegungen unberührt.

Eine Aufwertung tierischer Lautkommunikation ist epistemisch angezeigt. Von Descartes bis in den Behaviorismus wurde diese Dimension unterschätzt. Zu den pflanzlichen Signalcodes treten, wie gezeigt, im Tierreich phonetisch variable Kundgaben mit unterschiedlichen kommunikativen Funktionen auf. Diese über das Tierrech verteilte Fähigkeiten sind sicherlich graduell; sie reichen in ihren höchsten Formen an sprachliche Mitteilungen und Sprechhandlungen heran.

Wenn Lautbekundungen von Naturwesen einen kommunikativen, expressiven oder protosprachlichen Charakter haben und von Menschen, die die Rolle von advokatorischen Fürsprechern einnehmen, übersetzt bzw. versprachlicht werden, dann könnten diese Lautgaben hinlänglich für die Zuerkennung von Selbstwert sein. Dies gälte vor allem für Lautgaben, in denen sich Empfindungsfähigkeit und Kommunikation verschränken wie bei Schmerzenslauten, aber auch bei Expressionen von Lebensbejahung und -freude. Es ist denkbar, dass Kommunikations- und Empfindungsfähigkeit zwei korrelative Momente von Weltoffenheit sind, die im Reich der Lebewesen allmählich anwächst (Jonas 1973) und beim Menschen, der einen Weltbegriff zu bilden vermag, den Höhepunkt erreicht.

Für die Diskursethik sind Eigenschaften generell von besonderem Interesse, die Wesen zu Mitgliedern von unterschiedlichen realen $\mathrm{Ko} \mathrm{m} \mathrm{m} \mathrm{u}$ nikati on s g e me in sch aft en machen. Moralisch stark relevant wären Kommunikationsgemeinschaften aus Menschen und Tieren. Wenn es art- 
übergreifende Verständigungen gäbe (Mensch-Schimpanse, Mensch-Delphin, Mensch-Pferd usw.), so wäre in solchen Fällen eine in jedem Falle kommunikativ und/oder protosprachlich vermittelte Partnerschaft gegeben. In solchen kommunikativen Interaktionen, für die Interaktionen mit Haustieren paradigmatisch sind, nehmen Tiere eine (unvollständige) Du-Rolle ein (Habermas 1991: 220-224). In artübergreifenden Kommunikationen (Mensch-Schimpanse, Mensch-Delphin, Mensch-Pferd usw.), wäre dann eine Kommunikationspartnerschaft gegeben. Die Folgen sind weitreichender als man glaubt. Wenn kommunikativen Erfahrungen mit einzelnen Tieren der Spezies S plausibilisiert werden können, dann sind im Prinzip alle Exemplare dieser Spezies potentielle Kommunikationspartner mit Menschen, die solche Kommunikation über Artgrenzen hinweg vermögen. Es ist unerheblich, wie viele oder wenige dies sein werden. Den an solchen Interaktionen beteiligten nichtmenschlichen Partnern die Zuerkennung von Selbstwert zu verweigern, wäre speziesistisch. Es wäre dann auch eigenartig, Hunden und Katzen Selbstwert zuzuerkennen, ihren wild lebenden Verwandten wie etwa Wölfen und Tigern jedoch nicht. Aber wenn solche Kommunikationsgemeinschaften real bestehen, dann ist es nicht abwegig, auch hier das apelsche Prinzip der Beförderung von Idealität anzusetzen. Hierfür wären entsprechende Kommunikationspraktiken und -techniken zu entwickeln. Jamming with the whales wäre eine von vielen Möglichkeiten.

Hinzu kommt noch ein weiterer Gesichtspunkt: Gesetzt, die tierische Lautkommunikation sei eine notwendige naturhistorische Bedingung der Entstehung von Sprache gewesen. So, wie sich Selbstbewusstsein nicht ohne Bewusstsein hätte bilden können, so konnte sich die menschliche Sprache nur aus Lautkommunikation entwickeln (nicht jedoch aus biochemischer Signalübermittlung). Lautkommunikation ist die Vo rlä u fe rin der Sprache. Nun sind zwar nicht alle notwendigen Bedingungen selbstwerthafter Wesen ihrerseits selbstwerthaft. Sauerstoff ist notwendige Bedingung für viele Lebewesen, aber $\mathrm{O}_{2}$-Moleküle haben keinen Selbstwert. Bei geschichtlichen Vorläufern verhält es sich anders. Vorläufer leisten etwas in Bezug auf etwas, das verdienstlich oder wertvoll ist. Sie eröffnen und ebnen Wege, die zu etwas Neuem führen. Die Vorläufer der Sprache haben diesen Weg eröffnet. Sie haben inn in gewisser Weise „für uns" frei gemacht, ohne dies intendiert zu haben. Die Fähigkeit, eine Sprache zu sprechen, konnte nicht schlagartig entstehen; es bedurfte hierfür die Menschwerdung insgesamt. Deshalb bedeutet, hoch von Sprache und Diskurs zu denken, nicht, niedrig von Lautkommunikation zu denken. Vielmehr handelt sich, von uns aus betrachtet, um „de-potenzierte“ Sprache und vollwertige Kommunikation.

\section{Lösungsvorschlag}

Gesetzt, diese Unterscheidungsarbeit und die Zuordnungen seien plausibel. Was folgt daraus für das Inklusionsproblem? Gesetzt, wir könnten zur Lösung des Inklusionsproblems mehr als nur genau ein Kriterium heranziehen. Es 
kämen dann drei kriteriale Komplexe näher in Betracht: a) E m p f in d s a m keit, b) Interessenbezug, c) Kommunikationsfähigkeit. Jeder kriteriale Komplex kann nun in eine eher starke und eine eher schwache Variante unterteilt werden. Empfindsamkeit kann (stark) an das Vorliegen von Bewusstsein oder (schwach) an ein spürendes Gewahren gebunden werden. Interessen können (stark) als ein „Interessenhaben" oder (schwach) an ein "im-Interesse-sein" verstanden werden. Kommunikationsfähigkeit schließlich kann (stark) erst auf der Ebene von Lautkommunikation oder (schwach) bereits auf der Ebene von Informationsübertragung angesetzt werden. Die Ergebnisse auf allen drei Kriterien ähneln einander auf verblüffende Weise. In den drei starken Varianten wird moralischer Selbstwert allen Wirbeltieren zugesprochen, d.h. empfindungsfähigen Wesen, die ihre Interessen haben und - mit der bezeichnenden Ausnahme der Fische - zur Lautkommunikation fähig sind. Diese Wesen sind „weltoffen“ in dem Sinne, dass sie ihre artspezifischen Umwelten, ihre Artgenossen, Angehörige anderer Spezies bewusst wahrnehmen können. Plessner nannte diese tierische Daseinsweise „offene Positionalität" (Plessner 1928). Unterhalb dieser Wesen befindet sich ein fragwürdiger Bereich, nämlich der Seinsbereich der Spinnen, Käfer, Bienen, Libellen, Austern usw., in dem ein spürendes Gewahren stattfinden mag (Ott 2008).

Das Kriterium „Kommunikationsfähigkeit“ scheint nun im Falle der Pflanzen zu ähnlichen Problemen zu führen, die schon bei den Kriterien „Interesse“ und „Bewusstsein“ auftraten. Die Frage, ob Informationsübermittlung hinreichend ist für die Aufnahme in die moral community, ähnelt der Frage, ob „schwache Interessen“ ausreichend sind. An der Oberfläche scheinen alle drei Kriterien auf die Frage zuzulaufen, warum nicht die jeweils schwache Variante hinreichend für die Zuerkennung von moralischem Selbstwert sein soll.

Aus diskursethischer Perspektive legt sich nun folgende Interpretation des Inklusionsproblems nahe: Sprach- und Diskurswesen können ihr Verhalten an Gründen orientieren, weshalb sie genötigt sind, sich gemeinsam unter der Idee der Freiheit zu denken. Ihnen kommt daher „Würde“ zu, was ihrer Instrumentalisierung Grenzen setzt. Freie Wesen haben ein Recht, Rechte zu haben, das sich zu einem System der Menschen- und Bürgerrechte entfalten lässt. Empfindungsfähige, der lautlichen Kommunikation fähige und aktiv interessierte Wesen verdienen die Zuerkennung von moralischem Selbstwert. Diese Wesen bilden die Gruppe der eindeutigen moral patients. Die jeweils schwachen Versionen der drei Kriterien bilden bei großzügiger Deutung (lectio benignissima) die Gruppe der uneindeutigen oder grenzwertigen moral patients. Es ist keineswegs irrational, diesen Wesen moralischen Selbstwert zuzuschreiben, aber es ist auch nicht zwingend. In diesem Grenzbereich sind kann-Bestimmungen eine moralisch anständige pluralistische Lösung. Die starken Varianten sind u n h in t e rg e h b a r für die Bestimmung der moral community, die schwachen sind fakult a ti v. Dies trifft sich auch mit unseren vortheoretischen Intuitionen: Wir trauern um ein verstorbenes Haustier und finden es schade, wenn eine Gartenpflanze im Winter erfriert. 


\section{Anmerkungen}

1 Birch schwankte zwischen „kein Kriterium “ und „unterschiedliche Kriterien in unterschiedlichen Situationen“. Der ethische Skeptizismus schlägt bei Birch in einen Situationismus um, der unbefriedigend bleibt.

2 Auch die Informationsübertragung durch genomische Proliferation wäre dann aber eine Art von Kommunikation. Und es sind Kommunikations- qua Replikationsfehler, die evolutionär wirksam sind.

3 Der Terminus „Kommunikation“ ist somit einerseits Oberbegriff und qualifizierter Unterbegriff für diese Ebene.

4 Auf die sprachethischen Implikationen dieses Systems haben von Humboldt, Hönigswald, Habermas und ich selbst hingewiesen.

5 Diese Behauptung einzulösen, erforderte einen eigenständigen Aufsatz - oder ein ganzes Buch.

\section{Literatur}

Apel, Karl-Otto (1976), Transformation der Philosophie. Frankfurt a.M.: Suhrkamp 1976.

Baluška, František, Stefano Mancuso und Dieter Volkmann (Hrsg.) (2006), Communication in Plants. Berlin und Heidelberg: Springer.

Barbieri, Marcello (Hrsg.), Introduction to Biosemiotics: The New Biological Synthesis. Dordrecht: Kluver 2008.

Bennett, Jonathan (1967), Rationalität. Frankfurt a.M.: Suhrkamp.

Birch, Thomas (1993), „Moral Considerability and Universal Consideration“. Environmental Ethics 15, 4: 313-332.

Brandom, Robert B. (1994), Making it Explicit. Cambridge/Mass.: Harvard University Press.

Deacon, Terrence D. (2003), „Multilevel Selection in a Complex Adaptive System: The Problem of Language Origin“. In: Bruce H. Weber und David D. Depew (Hrsg.), Evolution and Learning. Cambridge/Mass.: MIT Press: 81-106.

Dryzek, John (1990), „Green Reason - Communicative Ethics for the Biosphere“. Environmental Ethics 12, 3: 195-210.

Fichtel, Claudia und Marta Manser (2010), „Vocal Communication in Social Groups“. In: Peter Kappeler (Hrsg.), Animal Behavior: Evolutions and Mechanism. Berlin und Heidelberg: Springer: 29-54.

Gardner, Allen R., Beatrix T. Gardner und Thomas E. Van Cantfort, (Hrsg.) (1989), Teaching Sign Language to Chimpanzees. Albany: State University of New York Press.

Gorke, Martin (2010), Eigenwert der Natur. Ethische Begründung und Konsequenzen. Stuttgart: Hirzel.

Gottschalk-Mazouz, Niels (2000), Diskursethik. Berlin: Akademie Verlag.

Habermas, Jürgen (1981), Theorie des kommunikativen Handelns. Frankfurt a.M.: Suhrkamp.

Habermas, Jürgen (1983), Moralbewußtsein und kommunikatives Handeln. Frankfurt a.M.: Suhrkamp. 
Habermas, Jürgen (1984), Vorstudien und Ergänzungen zur Theorie des kommunikativen Handelns. Frankfurt a.M.: Suhrkamp.

Habermas, Jürgen (1991), Erläuterungen zur Diskursethik. Frankfurt a.M.: Suhrkamp. Habermas, Jürgen (1992), Faktizität und Geltung. Frankfurt a.M.: Suhrkamp.

Hauser, Marc D. (2001), Wilde Intelligenz. Was Tiere wirklich denken. München: Hanser.

Hauser, Marc D., Noam Chomsky und Tecumseh W. Fitch (2002), „The Faculty of Language: What Is It, Who Has It, and How Did It Evolve?" Science 298, November 2002: 1569-1579.

Hendlin, Yogi und Konrad Ott (2016), „Habermas on Nature: A Postnormal Reading Between Moral Intuitions and Theoretical Restrictiveness“. Environmental Ethics 38, 2: 183-208.

Hoffmeyer, Jesper und Kalevi Kull (2003), „Baldwin and Biosemiotics: What Intelligence is for". In: Bruce H. Weber und David D. Depew (Hrsg.), Evolution and Learning. Cambridge/Mass.: MIT Press: 253-272.

Hönigswald, Richard (1937), Philosophie und Sprache. Basel: Drei Falken.

Humboldt, Wilhelm von (1979 [1829]), „Ueber die Verschiedenheiten des menschlichen Sprachbaues“. In: Werke, Bd. III, Darmstadt:Wissenschaftliche Buchgesellschaft: 144-367.

Jonas, Hans (1973), Organismus und Freiheit. Göttingen: Vandenhoek \& Ruprecht.

King, Stephanie L. und Vincent M. Janik (2013), „Bottlenose Dolphins Can Use Learned Vocal Labels to Address Each Other“. PNAS 110, 32: 13216-13221.

Mead, George H. (1973), Geist, Identität und Gesellschaft. Frankfurt a.M.: Suhrkamp.

Ott, Konrad (1993), Ökologie und Ethik. Tübingen: Attempto.

Ott, Konrad (1997), Ipso Facto. Zur ethischen Rekonstruktion normativer Implikate wissenschaftlicher Praxis. Frankfurt a.M.: Suhrkamp.

Ott, Konrad (2008), „A Modest Proposal of How to Proceed in Order to Solve the Problem of Inherent Moral Value in Nature“. In: Laura Westra, Klaus Bosselmann und Richard Westra (Hrsg.), Reconciling Human Existence with Ecological Integrity. London: Earthscan: 39-59.

Ott, Konrad (2010), Umweltethik zur Einführung. Hamburg: Junius.

Plessner, Helmut (1928), Die Stufen des Organischen und der Mensch. Berlin: De Gruyter.

Russon, Anne E., Kim A. Bard und Sue T. Parker (Hrsg.) (1996), Reaching into thought: The minds of the great apes. Cambridge und New York: Cambridge University Press.

Simmonds, Mark Peter (2006), „Into the Brains of Whales“. Applied Animal Behaviour Science 100: 103-116.

Tomasello, Michael (2012), „Von sozialer Interaktion zu sozialen Institutionen“. In: Julian Nida-Rümelin und Özmen Elif (Hrsg.), Welt der Gründe. Hamburg: Felix Meiner: 313-332.

Trewavas, Anthony (2014), Plant Behaviour and Intelligence. Oxford: Oxford University Press.

Werner, Micha (2003), Diskursethik als Maximenethik. Würzburg: Königshausen \& Neumann. 
Westerkamp, Dirk (2016), „Animal symbolica?“ In: Kristian Köchy, Matthias Wunsch und Martin Böhnert (Hrsg.), Maximen und Konsequenzen. Freiburg und München: Alber: 119-162.

Wittgenstein, Ludwig (1980), Philosophische Untersuchungen. Frankfurt a.M.: Suhrkamp.

\section{Prof. Dr. Konrad Ott}

Philosophisches Seminar der Christian-Albrechts-Universität zu Kiel

Leibnizstr. 6 / Zi. 316

$D-24118 \mathrm{Kiel}$

E-Mail: ott@philsem.uni-kiel.de 